\title{
A longitudinalidade e a integralidade no cuidado às crianças menores de um ano: avaliação de cuidadores*
}

\section{Longitudinality and comprehensive care of children under one year old: assessment of caretakers ${ }^{1}$}

\author{
Janaina C Braz¹, Débora F de Mello², Yan GM David³, Simone A Teixeira ${ }^{4}$, Aline Sousa Prado5 , Maria Cândida C
} Furtado ${ }^{6}$

\begin{abstract}
RESUMO
Objetivo: avaliar os atributos longitudinalidade e integralidade da atenção em Unidades de Saúde da Família em Vitória da Conquista, Bahia, Brasil. Método: pesquisa quantitativa, transversal com dados coletados mediante uso do Instrumento de Avaliação da Atenção Primária, versão nacional Primary Care Assessment Tool. Este instrumento contempla os atributos essenciais da APS, dentre eles a longitudinalidade e a integralidade da atenção. Foram entrevistados 271 cuidadores de crianças menores de um ano, usuários dos serviços, no período de janeiro a junho de 2012. Resultados: a longitudinalidade apresentou escore elevado $(3,07 \pm 0,53)$, demonstrando boa experiência dos cuidadores com as ações derivadas desse atributo. Por outro lado, os escores para integralidade, tanto para serviços disponíveis quanto para serviços prestados, foram baixos $(2,83 \pm 0,36$ e $2,81 \pm 0,98)$. Tais resultados apontam pouca percepção dos cuidadores sobre serviços disponíveis, como também serviços prestados à criança pelas unidades de saúde. Conclusões: A ESF constitui referência para a maioria das necessidades de saúde da criança, favorece a relação entre cuidadores e profissionais, além de possibilitar o conhecimento da situação de saúde desse público. Entretanto, apesar da alta cobertura da estratégia no município, algumas ações em saúde voltadas às crianças não são vivenciadas pelos cuidadores. Aos gestores e profissionais de saúde que atuam com esta clientela, cabe a sensibilização sobre as prioridades dentro de suas agendas, como também o planejamento e a implementação de ações que estejam dentro das necessidades de saúde da população infantil.
\end{abstract}

Palavras-chave: Atenção Primária à Saúde. Continuidade da Assistência ao Paciente. Assistência Integral à Saúde. Avaliação em Saúde. Cuidado da Criança.

1. Enfermeira. Mestre em Ciências. Doutoranda pelo Programa de Enfermagem em Saúde Pública da Escola de Enfermagem de Ribeirão Preto da Universidade de São Paulo (EERP-USP).

2. Professor Associado. Departamento de Enfermagem Materno-Infantil e Saúde Pública da EERP-USP.

3. Estudante de Medicina. Universidade do Sudoeste da Bahia (UESB).

4. Docente. Departamento de Ciências Naturais da Universidade do Sudoeste da Bahia (UESB)

5. Enfermeira do Centro de Imunizações Vacinnar.

6. Docente. Departamento de Enfermagem Materno-Infantil e Saúde Pública da EERP-USP.
Escola de Enfermagem de Ribeirão Preto da Universidade de São Paulo (EERP-USP). Av. dos Bandeirantes, 3900, Bairro Monte Alegre, CEP: 14040-902, Ribeirão Preto, SP, Brasil. Email: janacbraz@ hotmail.com

Artigo recebido em 20/06/2013 Aprovado para publicação em 28/11/2013

\footnotetext{
* Este artigo é parte da Dissertação de Mestrado intitulada "Avaliação da atenção às crianças menores de um ano na Estratégia Saúde da Família em um município da Bahia, sob a ótica dos cuidadores".
} 


\section{Introdução}

A garantia da oferta de serviços de saúde à população confere à Estratégia Saúde da Família (ESF) a reorganização e o fortalecimento da Atenção Primária à Saúde (APS), além de caracterizá-la como porta de entrada prioritária do sistema de saúde ${ }^{1}$, constitucionalmente fundada nos princípios da universalidade, acessibilidade, integralidade e equidade do Sistema Único de Saúde (SUS), tendo por atributos essenciais a acessibilidade, a longitudinalidade, a integralidade e a coordenação da atenção. ${ }^{1}$

Dos atributos da APS, o interesse no presente estudo se concentra na longitudinalidade e na integralidade do cuidado. O primeiro por possuir característica central desse nível assistencial, uma vez que se trata do acompanhamento do paciente, conferindo a continuidade e efetividade do tratamento, ao longo do tempo o que contribui para a implementação de ações de promoção e de prevenção de agravos de alta prevalência. Além disso, este atributo permite a produção de diagnósticos e tratamentos mais precisos, com redução de encaminhamentos desnecessários para especialistas e para realização de procedimentos de maior complexidade. ${ }^{1,2}$

No que se refere ao atributo integralidade da atenção, a sua relevância é estabelecida por se constituir em um leque de serviços disponíveis e prestados na atenção primária, de modo a proporcionar todos os cuidados de atenção à saúde. ${ }^{1}$ Nesse sentido, para o cuidado da criança na abordagem da integralidade, torna-se essencial a interação entre os serviços de saúde, da atenção básica à especializada, com apoio diagnóstico e terapêutico, como também nos níveis de maior complexidade, com o acompanhamento de toda a trajetória da criança. ${ }^{3,4}$ Ressalta-se, ainda, a promoção de ações voltadas à prevenção de agravos e assistência em intercorrências para melhoria da qualidade de vida da criança, contribuindo para que esta tenha condições para desenvolver todo o seu potencial. 3,5

Como modo de monitoramento dos princípios estabelecidos e que norteiam o cuidado da criança, a avaliação da assistência é recomendada. ${ }^{3}$ Assim, avaliar a atenção e a forma de organização da ESF na atenção à saúde da criança possibilita identificar a existência de aspectos positivos do cuidado e/ou a ocorrência de fragilidades, de modo a oferecer subsídios aos gestores e profissionais de saúde para promoção de melhorias das ações e das práticas oferecidas a este grupo. ${ }^{6,78}$ Nesse sentido, o presente estu- do objetivou avaliar os atributos ordenadores da APS, longitudinalidade e integralidade, voltadas às crianças menores de um ano.

\section{Material e Método}

Estudo transversal, conduzido no município de Vitória da Conquista, Bahia, tendo por referencial o modelo donabediano de avaliação. ${ }^{9,10}$

Com população local estimada de 306.866 habitantes, ${ }^{11}$ o município possui Gestão Plena do Sistema Municipal de Saúde e o setor saúde conta com um total de 303 estabelecimentos de saúde, sendo 201 pertencentes à rede pública (dois estaduais e 199 municipais) e 102, à rede privada. O setor de internações conta com 270 leitos em estabelecimentos públicos e 590 leitos em estabelecimentos privados. Sete estabelecimentos, entre público e privado, possuem atendimento em emergência pediátrica, sendo um deles o Hospital Municipal Esaú Matos que se encontra no rol dos hospitais Amigo da Criança. ${ }^{11}$

Quanto aos serviços de atenção primária, há cobertura de $63 \%$ em todo o município, sendo que a zona rural possui cobertura de $100 \%$. Do total de 36 equipes de saúde, 28 possuem a Estratégia de Saúde da Família, sendo que 13 equipes estão situadas na zona urbana e 15 na zona rural; oito equipes encontram-se alocadas em Unidade Básicas de Saúde (UBS). Além disso, o município conta ainda com quatro equipes de Núcleo de Apoio à Saúde da Família (NASF). ${ }^{12}$

As 13 equipes de ESF situadas na zona urbana constituíram campo de pesquisa, sendo que a imersão ocorreu entre 03 de janeiro e 27 de junho de 2012. Para tanto, houve treinamento prévio de 10 estudantes voluntários, provenientes dos cursos de Enfermagem, Medicina e Nutrição de duas instituições públicas e ensino superior do município, compondo o corpo de entrevistadores.

Participaram do estudo mães ou principais responsáveis pelo cuidado de crianças menores de um ano de idade pertencentes à área de abrangência de cada equipe de ESF. Os critérios de exclusão foram: crianças não pertencentes à área de abrangência da USF, que não utilizavam o SUS como principal serviço de atendimento em saúde e que não haviam sido atendidas, anteriormente, pela unidade de saúde, ou foram atendidas somente uma vez. Tais critérios possibilitaram contribuição dos entrevistados com a investigação, ao fornecerem sua opinião acerca das ações em saúde e dos serviços direcionados à criança. 
A coleta dos dados foi estabelecida, pelo pesquisador principal, em cinco dias úteis para cada USF em dois períodos, manhã ( 8 às 12 horas) e tarde (13 às 17 horas). A seleção dos participantes ocorreu a partir da presença na USF para atendimento da criança na sala de vacina, consultas de enfermagem ou médica agendadas, visto que a possibilidade de atendimentos não agendados pode apontar a satisfação de uma necessidade do usuário, dentro do sistema de saúde.

Para a coleta dos dados utilizou-se o Instrumento de Avaliação da Atenção Primária (Primary Care Assessment Tool PCA-Tool) desenvolvido nos Estados Unidos por Barbara Starfield e já validado no Brasil, ${ }^{13}$ voltado para os serviços de saúde infantil. O PCATool-infantil permite medir a presença e extensão dos atributos (essenciais e derivados) dos serviços de Atenção Primária às crianças e o grau de afiliação do usuário com o serviço de saúde. Mediante respostas tipo Likert, o instrumento constrói os escores de Atenção Primária, com intervalos que variam de 1 a 4 para cada atributo. $O$ escore final de cada atributo é a média das respostas de seus itens, que também variam de 1 a 4 , onde 4 representa maior presença e extensão do atributo e 1 a menor presença do mesmo. ${ }^{13,14}$

Os cuidadores foram abordados após o atendimento da criança na USF, com o intuito de não comprometer a rotina de trabalho da equipe local. Ao serem abordados e aceitarem participar do estudo, os mesmos foram entrevistados em lugar reservado, com sigilo das informações coletadas. O estudo foi aprovado por Comitê de Ética em Pesquisa (protocolo ${ }^{\circ}$ 1399/2011), sendo respeitados todos os preceitos éticos na condução do estudo.

Após as entrevistas, as variáveis longitudinalidade e integralidade do cuidado do PCATool foram registradas em planilha eletrônica de dupla entrada, posteriormente validada e, em seguida, os dados foram processados no Statistical Package for the Social Science (SPSS), versão 16.0. Calculou-se os escores para os atributos longitudinalidade e integralidade, dados pela média dos valores das respostas dos entrevistados. O escore obtido foi considerado elevado quando e" 3,0. ${ }^{14} \mathrm{~A}$ análise dos dados pautou-se na estatística descritiva e foi realizada à luz dos princípios do SUS e dos atributos da APS.

\section{Resultados}

Foram abordados, nas USF, 327 mães/cuidadores; destes, $271(81,4 \%)$ foram incluídos no estudo.
Dos 56 sujeitos restantes, registraram-se 13 recusas; 43 foram excluídos, uma vez que 19 eram responsáveis por crianças atendidas por convênios de saúde, cinco não se constituíam o cuidador principal e 19 crianças pertenciam à outra área de abrangência.

Do total de participantes do estudo, 257 (95\%) eram mães e $14(5 \%)$ responsáveis pelo cuidado da criança; optou-se por denominar todos os sujeitos entrevistados, como cuidadores. Das crianças cujos cuidadores responderam às entrevistas, $136(50,2 \%)$ eram do sexo feminino e $135(49,8 \%)$ do sexo masculino. A média da idade das crianças foi de 4,9 meses $(\mathrm{DP}=3,2)$.

A Tabela 1 apresenta os resultados da avaliação da atenção à criança menor de um ano com foco nos atributos Longitudinalidade e Integralidade, segundo cuidadores.

Para o atributo Longitudinalidade, o escore apresentado foi elevado $(3,7 \pm 0,53)$, sendo que os entrevistados reconhecem o mesmo profissional de saúde como responsável pelo seguimento da criança na USF. Porém, não consideram que poderiam ter suas dúvidas retiradas via ligação telefônica feita à unidade de saúde, caso fosse necessário.

Com relação à comunicação entre profissional de saúde e cuidador, estes reforçam que há uma estreita ligação que permite um diálogo claro entre ambos, com espaço para escuta, esclarecimento de dúvidas, o que os leva a sentirem-se confortáveis para colocar os questionamentos que possuem acerca do cuidado da criança. Outro aspecto positivo destacado diz respeito ao conhecimento que o profissional de saúde que acompanha a criança possui sobre a história clínica da mesma.

Como aspecto negativo, os cuidadores referiram que o profissional de saúde que atende a criança conhece-a melhor a partir do problema de saúde. Revelaram também, que a disponibilidade do profissional de saúde para conversar com toda a família a respeito da saúde da criança ainda não se constitui uma realidade.

Obteve-se baixo escore $(2,83 \pm 0,86)$ no atributo relacionado à Integralidade que representa a disponibilidade de serviços nas ESF. Dentre os serviços reconhecidos pelos cuidadores estão vacinação, participação em programas de assistência social ou benefícios sociais, planejamento familiar, realização de suturas em caso de cortes e aconselhamento e solicitação de teste anti-HIV. Entretanto, não foram identificados serviços como programa de suplementação nutricional, aconselhamento ou tratamento para uso de dro- 
Tabela 1: Valores dos escores dos atributos Longitudinalidade e Integralidade da atenção nas Unidades de Saúde da Família, zona urbana. Vitória da Conquista, 2012.

\begin{tabular}{lccccc}
\hline Atributos & Mínimo & Máximo & Média & DP \\
\hline Longitudinalidade & 1,8 & 4 & 3,07 & 0,53
\end{tabular}

- Quando você vai ao (USF de referência) é o mesmo médico ou enfermeiro que atende sua criança todas as vezes?

- Se você tiver uma pergunta sobre a saúde de sua criança, pode telefonar e falar com o médico ou enfermeiro que melhor conhece sua criança?

- Você acha que o médico ou enfermeiro da sua criança entende o que você diz ou pergunta?

- O médico ou enfermeiro responde suas perguntas de maneira que você entenda?

- O médico ou enfermeiro te dá tempo suficiente para falar sobre suas preocupações ou problemas?

- Você se sente a vontade contando suas preocupações ou problemas sobre sua criança ao médico ou enfermeiro?

- O medico ou enfermeiro conhece sua criança mais como pessoa ou somente alguém com um problema de saúde?

- O médico ou enfermeiro conhece a história clínica (médica) completa de sua criança?

- O médico ou enfermeiro sabe a respeito de todos os medicamentos que sua criança está tomando?

- O médico ou enfermeiro se reuniria com sua família se você achasse necessário para sua criança?

- Você mudaria do (USF de referência) para outro serviço de saúde se isto fosse muito fácil de fazer?

\section{Integralidade - Serviços disponíveis}

- Vacinas (imunizações).

- Planejamento familiar.

- Programa de suplementação nutricional (ex: leite e alimentos).

- Aconselhamento ou tratamento para o uso prejudicial de drogas (lícitas ou ilícitas: Álcool, remédios para dormir, cocaína, outros).

- Aconselhamento para problemas de saúde mental.

- Sutura de um corte que necessita de pontos.

- Aconselhamento e solicitação de teste anti-HIV?

- Identificação (algum tipo de avaliação) de problemas visuais (para enxergar).

\section{Integralidade - Serviços prestados}

- Orientação para manter sua criança saudável, como alimentação saudável, boa higiene ou sono adequado.

- Segurança no lar: como guardar medicamentos com segurança .

- Mudanças do crescimento e desenvolvimento da criança, isto é, que coisas você deve esperar de cada idade. Por exemplo, quando a criança vai caminhar, controlar o xixi,....

- Maneiras de lidar com o comportamento de sua criança.

- Maneiras para manter sua criança segura, como: Evitar tombos ou quedas de altura ou manter afastadas do fogão. 
gas ou para problemas de saúde mental e identificação de problemas visuais.

Para o atributo da Integralidade com foco em serviços prestados, o escore também foi baixo $(2,81 \pm 0,98)$. Apesar do valor apresentado, identificouse que a maioria dos entrevistados consideram que durante a consulta da criança, os profissionais de saúde abordam temas como segurança no domicílio, mudanças do crescimento e desenvolvimento da criança, ao mesmo tempo em que são fornecidas orientações sobre a manutenção da saúde da criança. Não foram identificadas, pelos cuidadores, orientações sobre maneiras para lidar com o comportamento das crianças e para mantê-las seguras.

\section{Discussão}

No presente estudo o cuidado ofertado à criança na ESF foi avaliado a partir dos atributos longitudinalidade e integralidade da atenção sob a ótica dos cuidadores. Tais sujeitos tornaram-se foco de interesse para este campo de investigação, uma vez que a visão avaliativa do usuário se faz importante dentro do processo assistencial, pois este utiliza o serviço de saúde e vivencia o cuidado ofertado com seus aspectos positivos e negativos. Nesse sentido, o serviço tende a ser avaliado de modo mais crítico pelo usuário do que pelos profissionais de saúde. ${ }^{8,15,16}$

Considerando os atributos da APS aqui investigados, os achados apontam que os aspectos da longitudinalidade foram satisfatórios, enquanto os da integralidade apresentaram-se frágeis. Diferentemente, um estudo comparativo entre os serviços de atenção primária, sob a ótica dos profissionais de saúde, evidenciou que para a ESF os escores dos atributos longitudinalidade e integralidade (serviços disponíveis) foram baixos, aproximando-se da média adequada. Já o escore de integralidade relativo aos serviços prestados foi alto. ${ }^{17}$

A longitudinalidade é considerada como atributo-chave da $\mathrm{APS}^{2,18}$ e os resultados dessa investigação corroboram a literatura, uma vez que as USF foram identificadas como ponto de partida para o cuidado da criança. Tal atributo está fortemente relacionado à boa comunicação que tende a favorecer o acompanhamento do paciente, a continuidade e efetividade do tratamento, e permite aos profissionais lançar mão de ações que promovam a saúde e previnam agravos. ${ }^{1,19}$

Ao se considerar cada aspecto que compõe o atributo Longitudinalidade, dentro do instrumento utili- zado nessa investigação, observou-se que a maioria apresentou altos valores de escores, o que resultou em escore elevado para este atributo como um todo, à semelhança de outros estudos. ${ }^{8,15,20}$ Entretanto, o estudo revela a permanência da centralidade da atenção na doença, o que contraria um dos objetivos centrais da ESF, que é a de reversão do modelo assistencial centrado na doença para um modelo centrado na promoção da saúde.

Desse modo, apesar da fragilidade de alguns aspectos, percebe-se que a ESF constitui referência para a maioria das necessidades de saúde da criança, favorece a relação entre cuidadores e profissionais de saúde, além de possibilitar o conhecimento da situação de saúde dessa clientela. Nesse sentido, a interação entre a equipe de saúde e a família e a história de vida da criança é imprescindível para uma assistência de qualidade. Resultados semelhantes foram encontrados em estudo ${ }^{20}$ que verificou a existência de vínculo entre pacientes e equipe de saúde, revelando que os responsáveis pelas crianças consideram satisfatória a postura dos profissionais.

Outro estudo ${ }^{19}$ ressalta a importância da qualidade da comunicação entre a família e os profissionais na assistência à saúde da criança para alcançar adequados resultados de saúde. Apontam a necessidade de a equipe repensar suas práticas para aumentar o vínculo com a população, a adesão às medidas de proteção e promoção à saúde, a atuação efetiva dos profissionais de saúde junto às famílias e a construção de planos de responsabilização e de projetos de saúde.

Uma das estratégias que tem levado as equipes de saúde a reorganizar o processo de trabalho é o acolhimento que, associado à presença de profissionais capacitados para uma escuta ativa e qualificada às suas demandas, possibilita a autonomia, a cidadania e a corresponsabilização na produção do cuidado à saúde. Assim, o acolhimento deve ser capaz de incluir os usuários nos serviços e, ao mesmo tempo, potencializar os profissionais de saúde e gestores na construção de espaços democráticos, éticos e reflexivos para a construção de um novo modelo assistencial, capaz de produzir sujeitos, cuidado e saúde. ${ }^{21,22}$

Em contrapartida, estudos realizados em diversas regiões do Brasil apresentaram resultados diferentes para o atributo da longitudinalidade no âmbito da ESF, com baixas médias de escores encontradas. ${ }^{16,23}$ Sendo assim, a avaliação da longitudinalidade é oportuna, posto que tal atributo é caracterizado como 
aspecto fundamental e exclusivo da APS e está relacionado à sua eficiência. ${ }^{1,2,18}$ Todavia, é importante considerar que um resultado positivo para tal atributo só será possível se o gestor de saúde priorizar em seu escopo esses serviços e ações, na medida em que envolvem questões como a oferta adequada de serviços de APS e mecanismos de fixação do profissional na unidade de saúde. ${ }^{2}$

No que se refere à Integralidade, observou-se escores baixos tanto para os serviços disponíveis quanto para os serviços prestados, diferente de outros estudos avaliativos. ${ }^{17,18} \mathrm{Tal}$ resultado aponta, a partir da experiência do cuidador, para ações de promoção e prevenção da saúde, voltadas ao cuidado integral à criança, não executadas. Uma possível explicação para tal fato pode ser a inexistência de alguns serviços e ações nas unidades, ou mesmo a falta de divulgação destes por parte dos profissionais de saúde.

Ressalta-se que todas as USF, cenários do estudo, possuem sala de vacina. Contudo, um pequeno percentual dos participantes relatou não haver tal serviço na unidade. Logo, o que se identifica é a existência de cuidadores que ainda desconhecem os serviços e ações ofertados pelas unidades de saúde. Também houve entrevistados que não identificaram a disponibilidade de alguns serviços na USF, como também destacaram temáticas não abordadas durante a consulta da criança, tais como os cuidados relacionados ao comportamento e à segurança da criança. Esses achados são semelhantes a estudo ${ }^{15}$ que identificou escores, para esses serviços e ações, aquém dos valores ideais.

Percebe-se que a assistência apresenta-se frágil, pois tais questões devem ser orientações de rotina, adaptadas a cada caso, considerando-se as especificidades de cada faixa etária para um melhor acompanhamento da criança nas consultas. Nesse aspecto, não somente os serviços disponíveis à população como um todo devem ser adequadamente divulgados, como também são necessárias as abordagens durante as consultas de acompanhamento do crescimento e desenvolvimento da criança. Tais serviços e temáticas de cuidado precisam chegar à população de modo que a mesma apreenda sua existência e compreenda sua importância.

Estudo $^{6}$ que teve por objetivo avaliar a atenção à criança pelo Programa Saúde da Família tendo por referência cenários distintos (incipiente, intermediário e avançado) evidenciou o despreparo dos profissionais de saúde acerca das medidas preventivas, de controle e de promoção da saúde da criança, com pouca participação dos profissionais, com exercício de práticas curativas oriundas do modelo biomédico, no âmbito da ESF. Para os autores tais fatos podem ser justificados pela sobrecarga de trabalho dos profissionais, cujos atendimentos são focados na queixa principal do paciente sem abordar outros aspectos do processo saúde-doença. ${ }^{6}$ Isso propicia a prática de atendimentos ineficazes e de baixa resolução, que estabelece obstáculos na construção de novas formas de produzir o cuidado na perspectiva da integralidade. ${ }^{6,23,24}$

Faz-se, portanto, necessária a reflexão acerca da oferta de serviços e do desenvolvimento de ações junto às crianças e sua família, uma vez que um bom atendimento e uma comunicação adequada entre paciente e profissional de saúde se constituem em ferramentas fundamentais para o desempenho eficaz da APS e devem ser oferecidos aos usuários dos serviços de saúde de forma indiscriminada. ${ }^{25}$

Dessa forma, é notório que fatores políticos, gerenciais, institucionais e estruturais limitam a integralidade do cuidado à criança na APS. Para alguns autores, ${ }^{24,26,27}$ a ESF não tem conseguido resolver os problemas de saúde da criança e da população em geral, e a articulação das ações entre a atenção primária, secundária e terciária acontece de modo informal, o que fere os princípios de acesso, equidade e integralidade da atenção definidos no SUS. Além disso, geram situações contínuas, como baixa resolutividade da APS, que contribui para o aumento da demanda nos demais níveis de complexidade, e consequentemente, eleva também a insegurança e a insatisfação da população com relação aos cuidados ofertados na APS. ${ }^{20,24,26}$

Diante disso, faz-se necessário que as unidades que atuam mediante a ESF ofertem e divulguem mais os serviços de saúde para a população, bem como disponibilizem profissionais capacitados para o cuidado infantil e que atendam às necessidades básicas e de grande impacto na saúde geral das crianças, famílias e comunidade. ${ }^{27}$ Para tanto, é necessário o empenho de trabalhadores e gestores da saúde, na construção de novas práticas direcionadas à saúde integral do paciente, com uma organização dos serviços de saúde estruturada a partir de um processo efetivo de educação permanente. ${ }^{17}$ 


\section{Conclusão}

O presente estudo revelou a importância da avaliação da saúde integral às crianças no âmbito da ESF, visto que seus resultados apontaram para ações positivamente aceitas e vivenciadas pelos cuidadores das crianças. Ao avaliar os atributos da APS, longitudinalidade e integralidade, os achados apontam que os aspectos da longitudinalidade foram satisfatórios, enquanto os da integralidade fragilizam a experiência do atendimento da criança no serviço de saúde.

O acompanhamento do crescimento e do desenvolvimento da criança, realizado pelo mesmo profissional de saúde, permite uma aproximação efetiva com os envolvidos no cuidado desta. Na continuidade dessa relação entre cuidador e profissional de saúde, torna-se possível o estabelecimento de um diálogo aberto. A partir daí, se inicia o conhecimento da criança enquanto indivíduo, não sendo foco do atendimento somente a queixa, a doença, o que permite transcender o cuidado para além do aspecto biológico.

Apesar da alta cobertura da ESF no município investigado, constata-se que nem todas as ações de saúde preconizadas pela APS estão sendo percebidas pelos cuidadores, no que se refere à assistência integral à saúde criança. Portanto, faz-se necessário o aprimoramento de serviços e ações que caracterizam a longitudinalidade e a integralidade, voltados ao cui- dado da criança, de modo que usuário, a partir de então, apreenda sua existência.

Os resultados oportunizam a retomada da força de cada um dos atributos avaliados. Na busca pelo cuidado qualificado à criança na Atenção Primária, é preciso que sejam disponibilizados tanto assistência com o mesmo profissional ao longo do tempo, quanto serviços diversificados e que ofereçam atendimento à saúde dessa clientela.

Aos gestores e aos profissionais de saúde que atuam diretamente com a criança, cabe a sensibilização sobre as prioridades dentro de suas agendas, como também o planejamento e a implementação de ações efetivas que estejam dentro das necessidades de saúde da população infantil.

As limitações desse estudo consistem no fato de os resultados serem restritos à zona urbana do município e sob a ótica apenas do cuidador. Isso implica que a avaliação à saúde da criança na APS precisa ser complementada por estudos que ofereçam a oportunidade para dar voz e permitir a reflexão também dos profissionais de saúde que atuam junto à criança $\mathrm{e}$ que, de perto, vivenciam o cotidiano do trabalho na ESF com suas potencialidades e/ou dificuldades. Além disso, as entrevistas foram realizadas nas unidades de saúde, o que pode restringir a generalização dos resultados às pessoas que utilizam tais serviços.

\begin{abstract}
Objective: This study aimed to evaluate the attributes longitudinality and comprehensive care in Family Health Units in Vitória da Conquista, Bahia, Brazil. Method: quantitative, cross-sectional study with data collected through the use of the Brazilian version of Primary Care Assessment Tool. This instrument covers the essential attributes of the Primary Health Care, including longitudinality and comprehensive care. We interviewed 271 caretakers of children under one year old who use the health facilities, from January to June 2012. Results: longitudinality scores were high (3.07 \pm 0.53$)$, demonstrating good experience of caretakers regarding the actions derived from this attribute. On the other hand, the scores for comprehensive care related to available services and services provided were low $(2.83 \pm 0.36$ and $2.81 \pm 0.98)$. Such results demonstrate little awareness of caretakers related to available services, as well as services provided to children by health facilities. Conclusions: Family Health Strategy is a reference for most health needs of the child; it promotes the relationship between caretakers and health professionals, and enables the knowledge about children health status. However, despite the high coverage of the Family Health Strategy in the county, some children's health actions are not experienced by caretakers. Managers and health professionals should have the priorities for child health in their agendas, as well as planning and implementation of actions which are related to the child health needs.
\end{abstract}

Key words: Primary Health Care, Continuity of Patient Care. Comprehensive Health Care. Health Evaluation. Child Care. 


\section{Referências Bibliográficas}

1. Starfield B. Atenção primária: equilíbrio entre necessidades de saúde, serviços e tecnologia. Brasília: UNESCO, Ministério da Saúde, 2002.

2. Cunha EM, Giovanella L. Longitudinalidade/continuidade do cuidado: identificando dimensões e variáveis para a avaliação da Atenção Primária no contexto do sistema público de saúde brasileiro. Ciênc Saúde Coletiva 2011; 16: 1029-42.

3. Ministério da Saúde. Secretaria de Atenção à Saúde. Departamento de Ações Programáticas Estratégicas. Agenda de compromissos para a saúde integral da criança e redução da mortalidade infantil. Brasília: Ministério da Saúde, 2004.

4. BRASIL. Portaria no 1459 de 24 de junho de 2011. Institui no âmbito do Sistema Único de Saúde-SUS a Rede Cegonha. 2011.

5. Furtado MCC, Mello DF, Parada CMGL, Pinto IC, Reis MCG, Scochi CGS. Avaliação da atenção ao recém-nascido na articulação entre maternidade e rede básica de saúde. Rev Eletrônica Enferm. 2010; 12: 640-6.

6. Costa G, Cotta MMR, Reis JR, Ferreira MLSM, Reis RS, Franceschini SCC. Avaliação da atenção à saúde da criança no contexto da Saúde da Família no município de Teixeiras, Minas Gerais. Ciênc Saúde Coletiva. 2011; 16: 3229-40.

7. Moreira MEL, Goldani MZ. A criança é o pai do homem: novos desafios para a área de saúde da criança. Ciênc Saúde Coletiva. 2010; 15:321-7.

8. Furtado MCC, Braz JC, Pina JC, Mello DF, Lima RAG. Assessing the care of children under one year old in Primary Health Care. Rev Latinoam Enferm. 2013; 21: 554-61.

9. Donabedian A. The quality of care - how come it be assessed? J Am Med Assoc. 1988; 260: 1743-8.

10. Donabedian A. La calidad de la atención médica: definición y métodos de evaluación. México: Ediciones Científicas La Prensa Médica Mexicana; 1984.

11. Instituto Brasileiro de Geografia e Estatística. Cidades: Vitória da Conquista, 2010. Disponível em: <http://www.ibge.gov.br/ cidadesat/topwindow.htm?1>. Acesso em: 17/05/2012.

12. Vitória da Conquista. Secretaria Municipal de Saúde de Vitória da Conquista. Dados do Município. Disponível em: <http:/ /www.pmvc.com.br>. Acesso em 10/05/2012.

13. Harzheim E, Starfield B, Rajmil L, Dardet CA, Stein AT. Consistência interna e confiabilidade da versão em português do Instrumento de Avaliação da Atenção Primária (PCATool Brasil) para serviços de saúde infantil. Cad Saúde Pública. 2006; 28: 1649-59.

14. Ministério da Saúde. Secretaria de Atenção em Saúde. Departamento de Atenção Básica. Manual do instrumento de avaliação da atenção primária à saúde: Primary Care Assessment Tool PCATool - Brasil / Ministério da Saúde, Secretaria de Atenção em Saúde, Departamento de Atenção Básica. - Brasília: Ministério da Saúde, 2010.
15. Leão CDA, Caldeira AP, Oliveira MMC. Atributos da atenção primária na assistência à saúde da criança: avaliação dos cuidadores. Rev Bras Saúde Matern Infant. 2011; 11: 32334.

16. Van Stralen CJ, Belisário AS, Van Stralen TBS, Lima, AMD, Massote AW, Oliveira CL. Percepção dos usuários e profissionais de saúde sobre atenção básica: comparação entre unidades com e sem saúde da família na Região CentroOeste do Brasil. Cad Saúde Pública. 2008; 24 (Supl.1): 14858.

17. Castro RCL, Knauth DR, Harzheim E, Hauser L, Duncan BB. Avaliação da qualidade da atenção primária pelos profissionais de saúde: comparação entre diferentes tipos de serviços. Cad Saúde Pública. 2012; 28: 1772-84.

18. Cassady CE, Starfield B, Hurtado MP, Berk RA, Nanda JP, Friedenberg LA. Measuring consumer experiences with primary care. Pediatrics. 2000; 105: 998-1003.

19. Mello DF, Furtado MCC, Fonseca LMM, Pina JC. Seguimento da saúde da criança e a longitudinalidade do cuidado. Rev Bras Enferm. 2012; 65: 675-9.

20. Ribeiro JM, Siqueira SAV, Pinto LFS. Avaliação da atenção à saúde da criança (0-5anos) no PSF de Teresópolis (RJ) segundo a percepção dos usuários. Ciênc Saúde Coletiva. 2010; 15:517-27.

21. Medeiros FA, Araújo-Souza GC de, Albuquerque-Barbosa AA, Clara-Costa IC. Acolhimento em uma Unidade Básica de Saúde: a satisfação do usuário em foco. Rev Salud Pública. 2010; 12: 402-13.

22. Mitre SM, Andrade EIG, Cotta RMM. Avanços e desafios do acolhimento na operacionalização e qualificação do Sistema Único de Saúde na Atenção Primária: um resgate da produção bibliográfica do Brasil. Ciênc Saúde Coletiva. 2012; 17:2071-85.

23. Mattioni FC, Budó M de LD, Schimith MD. O exercício da integralidade em uma equipe da estratégia saúde da família: saberes e práticas. Texto \& contexto enferm.. 2011; 20 263-71.

24. Sousa FGM, Edmann AL, Mochel EG. Condições limitadoras para a integralidade do cuidado à criança na atenção básica de saúde. Texto \& contexto enferm. 2011; 20: 263-71.

25. Mercado F, Mercado M, Myers N, Hewit M, Haller NA. Patient Preferences in Choosing a Primary Care Physician. J Prim Care Community Health. 2012; 3: 125-31.

26. Cardoso MO, Vieira-da-Silva LM. Avaliação da cobertura da atenção básica à saúde em Salvador, Bahia, Brasil (2000 a 2007). Cad Saúde Pública. 2012; 28: 1273-84.

27. Noronha MGRCS, Cardoso OS, Moraes TNP, Centa ML. Resiliência: nova perspectiva na promoção da saúde da família? Ciênc Saúde Coletiva. 2009; 14: 497-506. 